\title{
Finite element based system simulation for piezoelectric vibration energy harvesting devices
}

Journal of Intelligent Material Systems and Structures

2018, Vol. 29(7) 1333-1347

(C) The Author(s) 2017

Reprints and permissions:

sagepub.co.uk/journalsPermissions.nav DOI: 10.1 177/1045389X17733328

journals.sagepub.com/home/jim (SAGE

\section{Dominik Gedeon and Stefan J Rupitsch}

\begin{abstract}
We present a system simulation approach for piezoelectric vibration energy harvesting devices. Accurate modeling of the electromechanical structure is achieved by the finite element method. For consideration of power electronic circuits as a means of energy extraction, the finite element model is iteratively coupled to electric circuits via Simulink. The high computational cost of conventional finite element calculations is overcome by a specialized modal truncation method for general linear piezoelectric structures. In doing so, the simulation approach allows efficient prediction of mechanical quantities (e.g. displacements, stresses) as well as electric potentials in the continuum under the influence of arbitrary electrical circuits. Several examples are studied to validate the truncation approach against analytical models and full finite element models. The applicability of the method is demonstrated for a piezoelectric vibration energy harvester in conjunction with a power electronic circuit.
\end{abstract}

\section{Keywords}

Piezoelectric energy harvesting, power electronics, numerical simulation, finite element method, Simulink, model order reduction

\section{Introduction}

A vibration energy harvesting (VEH) device is an electromechanical structure along with an appropriate energy extraction circuit. It aims at converting otherwise unused mechanical energy present in vibrations of, for example, machinery to the electric domain. Aside from the piezoelectric effect, magnetostrictive (Wang and Yuan, 2008), electrostatic (Hoffstadt et al., 2013), or electromagnetic (Hendijanizadeh et al., 2013) conversion principles have been studied. Regardless of the energy conversion principle, the electromechanical structure has to be designed in accordance with the nature of the vibration source. Most commonly, laminated structures like unimorph or bimorph structures are employed in piezoelectric VEH for their exceptional efficiency when driven at resonance frequency (Beeby et al., 2013). Additional measures for increasing the bandwidth of resonant structures such as tuning of the resonance frequency by mechanical stresses $(\mathrm{Hu}$ et al., 2007; Wischke et al., 2010), magnetic forces (Challa et al., 2008), buckled beams (Van Blarigan et al., 2015), or centrifugal and Coriolis forces (Gedeon et al., 2014; $\mathrm{Gu}$ and Livermore, 2012) have been reported on. To predict the behavior of a piezoelectric $\mathrm{VEH}$, many authors offer a special modeling approach to their specific problem. In particular, lumped 1-degree-offreedom models or energy methods are often used for simple geometries. However, Erturk and Inman (2008b) have clarified some common misunderstandings in the context of piezoelectric vibration energy harvesters. They pointed out the inadequacy of simplistic 1-degree-of-freedom models for continua, as well as the errors in power estimation arising from negligence of piezoelectric coupling effects. Moreover, they suggested an analytical model for unimorph and bimorph cantilevered energy harvesters under the Euler-Bernoulli assumption (Erturk and Inman, 2008a). This modeling approach has extensively been applied throughout the literature on resonant VEH exploiting the direct piezoelectric effect. The underlying restriction to analytically solvable problems and their limited adaptability to changes in design or boundary conditions are

Chair of Sensor Technology, Friedrich-Alexander-Universität ErlangenNürnberg (FAU), Erlangen, Germany

\section{Corresponding author:}

Dominik Gedeon, Chair of Sensor Technology, Friedrich-AlexanderUniversität Erlangen-Nürnberg (FAU), Paul-Gordan-Straße 3/5, 91052 Erlangen, Germany.

Email: dominik.gedeon@fau.de 
shortcomings of analytical approaches. In contrast, design studies employing the finite element method (FEM) allow topology (Wein et al., 2013) or shape optimization (Park et al., 2012; Xiong and Oyadiji, 2014), assessment of different electrode geometries (Marqui et al., 2010), or detailed material parameter analyses (Daniels et al., 2013). Besides, parametric FE models offer a versatile means for the design and optimization of electromechanical structures under given boundary conditions and excitation scenarios. Compared to traditional approaches, FE simulations comprise, however, high computational effort.

In the case of piezoelectric conversion, extraction of energy as electric voltage and electric charge is particularly challenging, because of the high electrical impedance of piezoelectric materials at low vibration frequencies. When the design of the electromechanical structure is focused on, resistive electric loads are used in many cases for an initial assessment of extracted power. While estimation of maximum power output for an optimal electric load is useful for comparison between different harvester designs, optimal resistive loads do not reflect practical implementations of VEH devices. The most simple way of extracting electrical energy from a piezoelectric VEH is a diode bridge, supplying the rectified voltage to a capacitor or other energy storage elements. Thereby, controlled DC voltage converters are used to optimally adjust the voltage behind the rectifier (Kong and $\mathrm{Ha}, 2012$; Lefeuvre et al., 2007). Furthermore, specialized energy extraction methods, referred to as active extraction methods, have been subject to intensive research efforts (Deterre et al., 2012; Lefeuvre et al., 2009; Yiming et al., 2008). All of those practical extraction circuits include electrical switches as well as diode rectifiers or full-bridge inverters and control logic.

The ability to perform design iterations for the optimization of the electromechanical structure is a great advantage of finite element procedures. Anisotropic material behavior can be considered and realistic modeling of composite structures can be achieved. Direct coupling of a FE formulation to electrical networks, however, is only possible for linear time invariant (LTI) electrical networks or steady-state equivalent impedances of power electronics ( $\mathrm{Wu}$ and Shu, 2015). In order to combine the versatility of FE models with transient circuit simulations for nonlinear electric circuits, Elvin and Elvin (2009a) presented an explicit scheme for coupling of a commercial structural FE solver (for mechanics) to a circuit simulator. They achieved piezoelectric coupling to a lumped capacitance in the circuit simulator by parameters, which result from a RayleighRitz model. This approach combines the flexibility in geometrical design of the FEM with the powerful PSPICE engine. The method seems feasible to perform coupled simulations after subsequent simulation-based design of both the structural and the electronic part of the device. Nevertheless, the drawback of the FEM, namely high computational effort, impedes a combined optimization effort. Recently, Wu and Shu (2015) suggested steady-state equivalent electrical impedance models for relevant power electronic circuits with a FE model in COMSOL. The electrical steady-state impedances can be found in Shu and Lien (2006), Shu et al. (2007), and Lien et al. (2010). Yang and Tang (2009) developed an automated procedure to extract parameters for multi-modal equivalent circuit models (Elvin and Elvin, 2009b) from multiple FE analyses (including static, harmonic, and eigenfrequency computations). By doing so, the electromechanical structure is represented by an equivalent circuit model directly in PSPICE. Van Blarigan et al. (2015) extracted the geometry of cantilevers, that resulted from buckling by axial forces, from a FE analysis. Additionally, they computed linear mechanical mode shapes around the buckled equilibrium. With the help of the mode shapes, they approximated energy functions by analytical polynomials and derived equations of motion as well as piezoelectric coupling.

In this contribution, we propose an iteratively coupled simulation approach between a FE formulation with direct piezoelectric coupling and MATLAB/ Simulink. A directly coupled FE procedure ensures appropriate modeling of both the mechanical field and the electric field as well as their mutual coupling. The FE model is iteratively coupled to the multiphysics simulator Simscape via Simulink. Simscape provides electric circuit simulation capabilities, while traditional Simulink blocks for, for example, control design or logics are available as well. In Simulink, one is able to use implicit variable-step-solvers for time integration. Such implicit schemes are generally favorable over explicit schemes when dealing with differential algebraic equations, which arise from constraint electrical networks. Furthermore, we tackle the aforementioned problem of computational efficiency by a modified modal truncation technique for piezoelectrically coupled FE formulations. We highlight the benefits of our modified truncation method by comparisons to traditional mechanical truncation methods with lumped electric parameters, revealing some of their shortcomings. The applied procedures are demonstrated by means of a piezoelectric unimorph cantilever that is equipped with a power electronic circuit for active energy extraction.

\section{Piezoelectrically coupled FEM}

The FEM is a numerical procedure for the computation of boundary value problems, which are given by partial differential equations (PDEs) and boundary conditions (BCs) (Kaltenbacher, 2015). Such mathematical problems are not generally solvable by analytical means. In 
the case of piezoelectric materials, the PDEs for the mechanical field and for the electrostatic field are coupled by the piezoelectric effect. The mechanical field is described by Navier's equation

$$
f_{\mathrm{V}}+\nabla \cdot[\boldsymbol{\sigma}]=\rho \boldsymbol{a}
$$

where $f_{\mathrm{V}}$ stands for external volume forces and $\rho \boldsymbol{a}$ is the vector of inertial forces (material density $\rho$ and acceleration $\boldsymbol{a}$ ). The dot product of the Nabla Operator $\boldsymbol{\nabla}$ and the Cauchy stress tensor $[\boldsymbol{\sigma}]$ denotes the divergence of the material stresses.

Assuming electrically insulating material and neglecting electromagnetic wave phenomena, Maxwell's equations for the electric field reduce to

$$
\begin{gathered}
\boldsymbol{\nabla} \cdot \boldsymbol{D}=q_{\mathrm{e}} \\
\boldsymbol{E}=-\boldsymbol{\nabla} V_{\mathrm{e}}
\end{gathered}
$$

with the electric flux density $\boldsymbol{D}$, the electric charge density $q_{\mathrm{e}}$ on the electrodes, the electric potential $V_{\mathrm{e}}$, and the electric field $E$, respectively. Equations (1) to (3) are coupled by the constitutive equations of linear piezoelectricity (transpose $\mathrm{T}$ )

$$
\begin{gathered}
\boldsymbol{\sigma}=\left[\boldsymbol{c}^{\boldsymbol{E}}\right] \boldsymbol{S}-[\boldsymbol{e}]^{\mathrm{T}} \boldsymbol{E} \\
\boldsymbol{D}=[\boldsymbol{e}] \boldsymbol{S}+\left[\boldsymbol{\varepsilon}^{\boldsymbol{S}}\right] \boldsymbol{E}
\end{gathered}
$$

$\boldsymbol{\sigma}$ and $\boldsymbol{S}$ represent the vectors of mechanical stress and strain in Voigt notation. $\left[\boldsymbol{c}^{\boldsymbol{E}}\right],[\boldsymbol{e}]$, and $[\boldsymbol{\varepsilon}]$ are the material tensors for mechanical stiffness at constant electric field, piezoelectric moduli, and electric permeability at constant mechanical strain, respectively. Combining equations (1) to (5), we arrive at the coupled PDEs for piezoelectric materials in Voigt notation

$$
\begin{aligned}
& \rho \ddot{\boldsymbol{u}}-\mathbf{B}^{\mathrm{T}}\left(\left[\boldsymbol{c}^{\boldsymbol{E}}\right] \mathbf{B} \boldsymbol{u}-[\boldsymbol{e}]^{\mathrm{T}} \boldsymbol{\nabla} V_{\mathrm{e}}\right)=\boldsymbol{f}_{\mathrm{V}} \\
& \boldsymbol{\nabla} \cdot\left([\boldsymbol{e}] \mathbf{B} \boldsymbol{u}-\left[\boldsymbol{\varepsilon}^{\boldsymbol{S}}\right] \boldsymbol{\nabla} V_{\mathrm{e}}\right)=q_{\mathrm{e}}
\end{aligned}
$$

Here, $[\boldsymbol{\sigma}]$ has been substituted by $\left[\boldsymbol{c}^{\boldsymbol{E}}\right] \boldsymbol{S}$ and $\boldsymbol{S}=\mathbf{B} \boldsymbol{u}$ is expressed by the B-Operator and the displacement vector $\boldsymbol{u}$. The B-Operator realizes the divergence in Voigt notation. In the FEM, equations (6) and (7) are converted into their variational form. Subsequently, the computation domain is subdivided into finite elements and integration is performed over each element. The results of the spatial integration procedure are assembled into matrices that are (for the three-dimensional mechanical case) of the dimension $3 N_{\text {Nodes }} \times 3 N_{\text {Nodes }}$ and sparse. $N_{\text {Nodes }}$ is the number of finite element nodes, used to discretize the geometry. After applying the FEM with nodal finite elements, we arrive at a system of coupled ordinary differential equations (ODEs) for the unknown nodal displacements and electric potentials

$$
\begin{gathered}
\overbrace{\left(\begin{array}{cc}
\mathbf{0} & \mathbf{0} \\
\mathbf{0} & \mathbf{M}_{\mathbf{u}}
\end{array}\right)}^{\mathbf{M}}\left(\begin{array}{c}
\ddot{\boldsymbol{V}} \\
\ddot{\boldsymbol{u}}
\end{array}\right)+\overbrace{\left(\begin{array}{cc}
\mathbf{0} & \mathbf{0} \\
\mathbf{0} & \mathbf{C}_{\mathrm{u}}
\end{array}\right)}^{\mathbf{C}}\left(\begin{array}{c}
\dot{\boldsymbol{V}} \\
\dot{\boldsymbol{u}}
\end{array}\right) \\
+\underbrace{\left(\begin{array}{cc}
-\mathbf{K}_{\mathrm{V}} & \mathbf{K}_{\mathrm{Vu}} \\
\mathbf{K}_{\mathrm{Vu}}^{\mathrm{T}} & \mathbf{K}_{\mathrm{u}}
\end{array}\right)}_{\mathbf{K}} \underbrace{\left(\begin{array}{c}
\boldsymbol{V} \\
\boldsymbol{u}
\end{array}\right)}_{\boldsymbol{x}}=\underbrace{\left(\begin{array}{c}
\boldsymbol{Q} \\
\boldsymbol{F}
\end{array}\right)}_{\boldsymbol{R}}
\end{gathered}
$$

$\mathbf{M}, \mathbf{C}$, and $\mathbf{K}$ are the mass, damping, and stiffness matrix, respectively. Indices $u$ indicate mechanical submatrices and a subscript $\mathrm{V}$ denotes the electric part of the equations. $\mathrm{Vu}$ denotes the coupling terms between mechanical and electric field. The mechanical damping matrix $\mathbf{C}_{u}$ consists of a mass- and stiffness-proportional fraction. The vector $\boldsymbol{x}$ contains the unknown nodal quantities, consisting of mechanical displacements $\boldsymbol{u}$ and electric potentials $\boldsymbol{V} . \boldsymbol{R}$ is the right-hand-side vector with the external electric charges $\boldsymbol{Q}$ and the external forces $\boldsymbol{F}$. Each dot above a variable indicates a single differentiation with respect to time.

Equation (8) is discrete in the spatial dimensions but still continuous in time. It can be solved in the timedomain by an appropriate time-integration scheme, or in the frequency domain by employing the Fourier transform of the time-derivatives. The accuracy of the method is strongly dependent on spatial discretization. A sufficient number of elements must be used in each geometric dimension to guarantee convergence toward the exact solution. The adequate number of elements can, for example, be reduced using hierarchic polynomial interpolation functions (p-FEM) or by making certain a-priori assumptions (Bathe, 2006; Kaltenbacher, 2015). In the following examples, we will only employ standard nodal (Lagrangian) elements with quadratic interpolation functions. We use a custom FE code CFS++ (Coupled Field Simulation in $\mathrm{C}++$ ) that was developed at the Chair of Sensor Technology (Kaltenbacher, 2010) for the computation and assembling of equation (8).

\section{Coupling of piezoelectric FEM with circuit simulation}

Piezoelectric devices consist of a structure, incorporating piezoelectric material and electronic circuitry. However, the system of coupled ODEs in equation (8) describes a piezoelectric transducer (i.e. sensor, actuator, or energy converter) without any exterior electronics. In some applications, both parts of the device might be considered independent from each other, that is, they do not exert significant mutual influence. This is, for example, the case, when piezoelectric coupling in the structure is weak. Then, the electromechanical structure behaves electrically like a capacitance and may be modeled as such in a circuit simulation environment. Sensors operating in open-circuit mode (measurement 
with very high resistance) or in short-circuit condition (measurement with a charge amplifier) constitute another scenario, where closed-loop coupling might be unnecessary. In those cases, the model output of the electromechanical simulation can serve as input for a circuit simulation without any feedback. If, however, significant exchange of electric power between circuit and structure occurs and the electromechanical coupling is strong, both parts of the system must be modeled accurately. Additionally, full coupling of electromechanical and circuit simulation is indispensable. The practical consequence of this finding is that electric current (or charge in our formulation (8)), which is drawn from the piezoelectric structure, must be fed back to the right-hand-side $R$ to account for its effects on voltage and mechanical displacements. In the following, we will propose a method to consider arbitrary electric loads in the context of a FE simulation in the time-domain.

\section{Coupling in Simulink for transient simulations}

It is possible to couple equation (8) directly with a set of ODEs that represent a passive electrical network. Generally, such approach exhibits two drawbacks: (1) a new set of equations needs to be deduced; each time a change in the network's topology is made and (2) the approach is restricted to LTI networks or steady-state representations of power electronics (Lien et al., 2010; Shu et al., 2007; Shu and Lien, 2006). When pursuing a system simulation approach, however, the need for more versatile simulation methods arises. To illustrate this fact, we will present an example for power electronic circuits including nonlinear electrical elements and time-variant network topology in section “Application example."Elvin and Elvin (2009a) developed an explicit scheme, coupling a structural (purely mechanical) FEM with a circuit simulator. They exploited the displacement results from a commercial FE software at each time step and derived an electric capacitance as well as a piezoelectric coupling factor. The displacement results from the FE software are coupled to the capacitance through the coupling term. Moreover, the piezoelectric nodal forces from the circuit simulator (caused by the electric current) are considered in the next time step of the FE calculation. Given a sufficiently small time increment, the solution converges. As already stated in Elvin and Elvin (2009a), an explicit algorithm is, however, not the most efficient procedure for coupled problems.

In contrast to the approach of Elvin and Elvin, let us directly use the system of ODEs in equation (8) for an implementation in Simulink. For this purpose, the equations for the mechanical and the electrostatic field are rearranged as follows

$$
\ddot{\boldsymbol{u}}=\mathbf{M}_{\mathrm{u}}^{-1}\left(\boldsymbol{F}-\mathbf{C}_{\mathrm{u}} \dot{\boldsymbol{u}}-\mathbf{K}_{\mathrm{u}} \boldsymbol{u}-\mathbf{K}_{\mathrm{Vu}}^{\mathrm{T}} \boldsymbol{V}\right)
$$

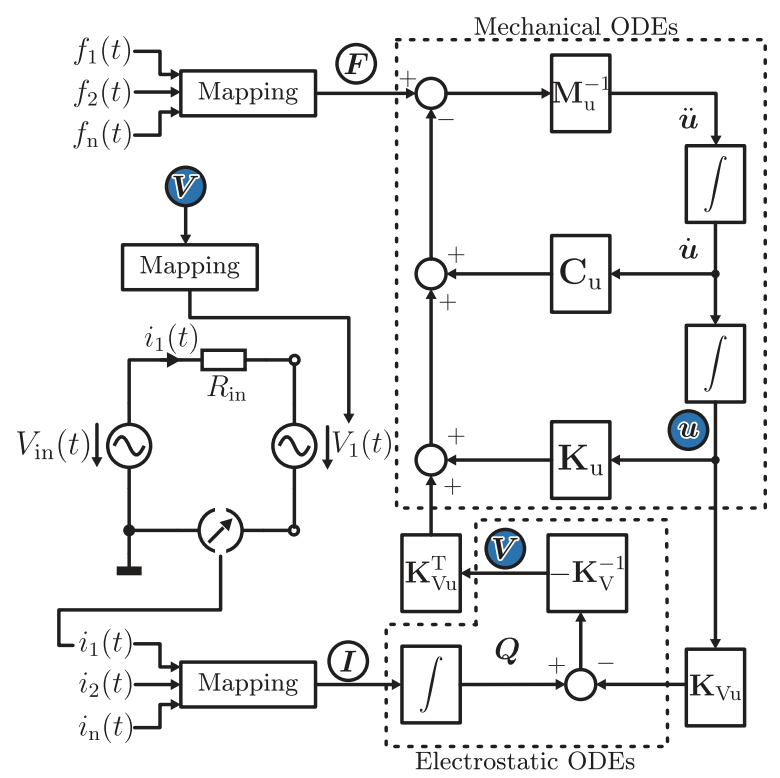

Figure I. Drawing of the implementation of equations (9) and $(10)$ as block diagram in Simulink. Electrical circuits are implemented through the Simscape library and coupled to the ODEs by controlled voltage sources and current measurements.

$$
\boldsymbol{V}=-\mathbf{K}_{\mathrm{V}}^{-1}\left(\boldsymbol{Q}-\mathbf{K}_{\mathrm{Vu}} \boldsymbol{u}\right)
$$

The structure of the corresponding block diagram is shown in Figure 1. External forces and currents (derivatives of the charges) can be applied to single nodes or regions/electrodes, which are mapped to the appropriate equation numbers in the system of ODEs. Figure 1 also illustrates the principle of connecting the piezoelectric device to an electrical circuit. We use the circuit simulation capability provided by the Simscape package to build an electrical circuit (consisting of a voltage source $V_{\text {in }}(t)$ with internal resistance $R_{\text {in }}$ in Figure 1) and consider the voltage drop over the piezoelectric element $V_{1}(t)$ by a controlled voltage source. The electric loop current is measured, integrated (to get the electric charge $Q(t)$ ), and mapped to the right-hand-side vector of the piezoelectric system of ODEs. Moreover, flexible interconnections between electrodes of bimorph or segmented-electrode structures can be achieved, considering all capacitive and electromechanical coupling effects between electrical terminals. The vector of nodal displacements $\boldsymbol{u}$ is available for displaying or postprocessing (e.g. calculation of material stresses). Modeling of the electrical network can also be achieved by implementing the network's equations by traditional Simulink blocks such as transfer functions and statespace representation.

\section{Modal truncation}

The implemented coupled FE system in Figure 1 generally involves a large number of coupled equations, that 
is, one equation for each mechanical degree of freedom plus one equation for each nodal electric potential. The sparsity of the FE matrices cannot be exploited, since Simulink does not provide sparse algebra. This implies that all matrices are stored fully populated into memory. Accordingly, one can only solve FE models with very limited degrees of freedom only. The disadvantage of relatively high computational cost in transient calculations persists for all hybrid methods involving the FEM. The modal truncation technique introduced in this section is a means of extracting relevant information from a full FE model while significantly reducing the mathematical size of the problem. It enables computationally efficient simulations for piezoelectric structures, connected to electronic circuits. Below, we will show that important results, usually extracted from the solution of a full FE problem, are reproduced accurately by our method and are readily obtained from the solution to the truncated problem.

\section{Modal truncation of mechanical systems}

The technique of modal truncation is based on mode superposition for mechanical dynamic FE analysis (Bathe, 2006). Briefly, the idea is changing the basis of the system of equations using a transformation matrix $\boldsymbol{\Phi}_{\mathrm{u}}$. Thereby, the nodal displacements $\boldsymbol{u}$ are expressed in generalized coordinates $\boldsymbol{u}^{*}$ and the underlying transform reads as

$$
\boldsymbol{u}(t)=\boldsymbol{\Phi}_{\mathrm{u}} \boldsymbol{u}^{*}(t)
$$

The transformation matrix must be non-singular in order to provide a unique mapping. With equation (11), the system of mechanical equations becomes

$$
\begin{gathered}
\boldsymbol{\Phi}_{\mathrm{u}}^{\mathrm{T}} \mathbf{M}_{\mathrm{u}} \boldsymbol{\Phi}_{\mathrm{u}} \ddot{\boldsymbol{u}}^{*}(t)+\boldsymbol{\Phi}_{\mathrm{u}}^{\mathrm{T}} \mathbf{C}_{\mathrm{u}} \boldsymbol{\Phi}_{\mathrm{u}} \dot{\boldsymbol{u}}^{*}(t) \\
+\boldsymbol{\Phi}_{\mathrm{u}}^{\mathrm{T}} \mathbf{K}_{\mathrm{u}} \boldsymbol{\Phi}_{\mathrm{u}} \boldsymbol{u}^{*}(t)=\boldsymbol{\Phi}_{\mathrm{u}}^{\mathrm{T}} \boldsymbol{F}(t)
\end{gathered}
$$

It is convenient to build the transformation matrix from the eigenvectors of the undamped free-vibration problem (i.e. assuming $\mathbf{C}_{\mathrm{u}}=0$ and $\boldsymbol{F}=0$ ) of the system, which is formulated as

$$
\mathbf{K}_{\mathrm{u}} \boldsymbol{\Phi}_{\mathrm{u}}=\boldsymbol{\Omega}^{2} \mathbf{M}_{\mathrm{u}} \boldsymbol{\Phi}_{\mathrm{u}}
$$

The matrix $\boldsymbol{\Phi}_{\mathrm{u}}$ consists of the eigenvectors that correspond to the mode shapes of the free-vibration problem. The matrix $\boldsymbol{\Omega}^{2}$ is a diagonal matrix, containing the corresponding eigenvalues, that is, the squared angular frequencies of the vibration. Since the eigenvectors are orthonormal with respect to the mass matrix $\mathbf{M}_{\mathrm{u}}$, the transformed mass matrix becomes the identity matrix, and the transformed stiffness matrix is equal to $\mathbf{\Omega}^{2}$. The damping matrix is a weighted superposition of mass and stiffness matrix (Rayleigh damping). As all matrices are diagonal, the equations are decoupled. In Elvin and Elvin (2009b), this approach is combined with a Rayleigh-Ritz modeling methodology to derive equivalent electric circuits of simple topology for mechanical equations.

Since in technical applications, the bandwidth of interest is often limited, the size of the system of equations can be reduced drastically by considering only the first $N$ mode shapes. The reduced system of equations is then valid up to frequencies in the range of $\omega_{\mathrm{N}}$. We refer to this practice as modal truncation because the system of equations is truncated after the $N$ th mode. When $N$ eigenvectors are considered for the transformation matrix $\boldsymbol{\Phi}_{\mathrm{u}}$, the transformed matrices reduce to a size of $N \times N$. The solution vector $\boldsymbol{u}^{*}(t)$ are the generalized displacements (i.e. defined in the modal subspace) and have to be transformed through equation (11) in order to obtain the displacements in Cartesian coordinates. It should be noted that the eigenvalue problem (13) is solved by iterative methods (e.g. Arnoldi Iteration (Lehoucq and Sorensen, 1996)). Hence, it is not necessary to compute all eigenvectors, when only the first $N$ vectors are considered in the transformation. This renders the technique computationally efficient.

\section{Modal truncation of piezoelectric systems}

When dealing with piezoelectrically coupled systems, the electrostatic field, the mechanical field as well as their mutual coupling have to be modeled. Most commonly, the electric part of the system is described by a lumped capacitance, which is then coupled by a distinct factor to each of the mechanical modes that are considered in the model. This method has been used in various studies with different means of calculating mechanical mode shapes and deriving the actual equations of motion (Elvin and Elvin, 2009a, 2009b; Erturk and Inman, 2008a, 2008b; Marqui et al., 2010; Van Blarigan et al., 2015; Yang and Tang, 2009). We denote this method by mechanical reduction with lumped electric parameters $(M L)$.

In this contribution, we propose a different, more accurate way of forming a reduced piezoelectric model. It does not lump the electric parameter, but considers the distribution of electric potentials within the material. It also considers piezoelectric coupling already at the stage of computing the mode shapes. We denote this method by coupled reduction with distributed electric parameters $(C D)$. Both types of modal reduction are drawn in Figure 2 and shall be further described in the following sections.

Mechanical reduction with lumped electric parameters (ML). The method, described in section "Modal truncation of mechanical systems," can be applied to the mechanical part of the system in order to obtain a reduced set of ODEs that describe the mechanical 


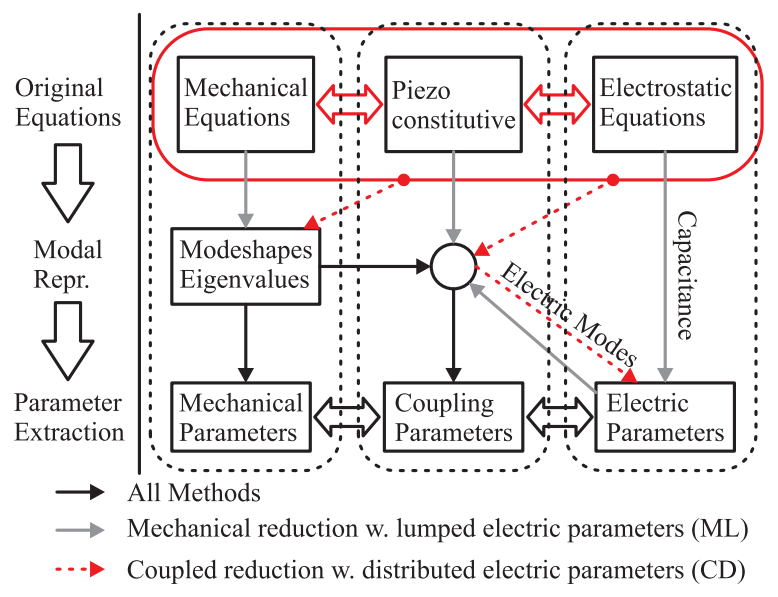

Figure 2. Scheme of modal truncation procedure and model parameter extraction for the reduced model. Gray arrows represent the traditional approach for piezoelectric systems. The red arrows indicate our modified approach, considering coupling within the original system of equations and providing distributed electric parameters.

behavior of the structure. In Figure 2, this is represented by the path from the original mechanical equations straight down to the extracted parameters. By original equations, we mean the starting point of model deduction, that is, for the mechanical part Euler-Bernoulli equations or, as in our case, the Navier equation (1) with subsequent FE discretization. The electric equation (3) is reduced to a capacitance between the hot electrode and electrical mass, which already comprises the electrical parameter in Figure 2. In the case of simple rectangular geometries, this can be achieved by the plate capacitor approximation, instead of a FEM calculation

$$
C_{\text {Plate }}=\varepsilon_{33} \frac{A}{d}
$$

Coupling between the electric and the mechanical part of the reduced system is then enabled by appropriate coupling factors, relating modal displacement and its corresponding charge on the capacitance. A modal coupling factor represents the electric charge on the lumped capacitance when the displacement of the mechanical structure equals a corresponding mode shape. Electromechanical coupling is not considered until after reduced parameter extraction. Consequently, the mechanical mode shapes are those of the uncoupled structure. Although in this article, we use the FEM for mechanical as well as electrostatic modeling, the mechanical mode shapes can also be derived from an analytical formulation (where solvable) (Erturk and Inman, 2008a) or other numerical methods (Elvin and Elvin, 2009a). In Elvin and Elvin (2009b), a full FE model is used instead of a truncated one. Nonetheless, the methodology of coupling the mechanical field to a lumped capacitance is the same as in Figure 2.
Table I. Mathematical procedure of the modal truncation for piezoelectric structures.

$$
\begin{aligned}
& \text { Solve } \\
& \mathbf{K \Phi}=\mathbf{\Omega}^{2} \mathbf{M \Phi} \quad \text { For } N \text { eigenvectors } \\
& \text { Solve } \\
& \phi_{\mathrm{p}}=\mathbf{K}^{-1} \boldsymbol{R} \\
& \text { Build } \\
& \boldsymbol{\Phi}_{\mathrm{u}}=\left(\phi_{\mathrm{ul}}, \ldots, \phi_{\mathrm{uN}}, \phi_{\mathrm{up}}\right) \quad \boldsymbol{\Phi}_{\mathrm{u}} \in \mathbb{R}^{\mathrm{N}_{\mathrm{u}} \times(\mathrm{N}+\mathrm{l})} \\
& \boldsymbol{\Phi}_{\mathrm{V}}=\left(\phi_{\mathrm{V} l}, \ldots, \phi_{\mathrm{VN}}, \phi_{\mathrm{V}_{\mathrm{p}}}\right) \quad \boldsymbol{\Phi}_{\mathrm{V}} \in \mathbb{R}^{\mathrm{N}_{\mathrm{V}} \times(\mathrm{N}+\mathrm{I})} \\
& \text { Compute } \\
& \mathbf{M}_{\mathrm{u}}^{*}=\boldsymbol{\Phi}_{\mathrm{u}}^{\top} \mathbf{M}_{\mathrm{u}} \boldsymbol{\Phi}_{\mathrm{u}} \\
& \mathbf{C}_{\mathrm{u}}^{*}=\boldsymbol{\Phi}_{\mathrm{u}}^{\top} \mathbf{C}_{\mathrm{u}} \boldsymbol{\Phi}_{\mathrm{u}} \\
& \mathbf{K}_{\mathrm{u}}^{*}=\boldsymbol{\Phi}_{\mathrm{u}}^{\top} \mathbf{K}_{\mathrm{u}} \boldsymbol{\Phi}_{\mathrm{u}} \\
& \mathbf{K}_{\mathrm{V}}^{*}=\boldsymbol{\Phi}_{\mathrm{V}}^{\top} \mathbf{K}_{\mathrm{V}} \boldsymbol{\Phi}_{\mathrm{V}} \\
& \mathbf{K}_{\mathrm{Vu}}^{*}=\boldsymbol{\Phi}_{\mathrm{V}}^{\top} \mathbf{K}_{\mathrm{Vu}} \boldsymbol{\Phi}_{\mathrm{u}} \\
& \boldsymbol{F}^{*}=\boldsymbol{\Phi}_{u}^{\top} \boldsymbol{F} \\
& \mathbf{Q}^{*}=\boldsymbol{\Phi}_{V}^{\top} \mathbf{Q} \\
& \text { Solve reduced problem } \\
& \text { Compute } \\
& \mathbf{u}=\boldsymbol{\Phi}_{\mathrm{u}} u^{*}, \boldsymbol{V}=\boldsymbol{\Phi}_{\vee} \boldsymbol{V}^{*} \\
& \mathbf{M}_{\mathrm{u}}^{*} \in \mathbb{R}^{(\mathrm{N}+\mathrm{I}) \times(\mathrm{N}+\mathrm{I})} \\
& \mathbf{C}_{\mathrm{u}}^{*} \in \mathbb{R}^{(\mathrm{N}+\mathrm{I}) \times(\mathrm{N}+\mathrm{I})} \\
& \mathbf{K}_{\mathrm{u}}^{*} \in \mathbb{R}^{(\mathrm{N}+\mathrm{I}) \times(\mathrm{N}+\mathrm{I})} \\
& \mathbf{K}_{\mathrm{V}}^{*} \in \mathbb{R}^{(\mathrm{N}+\mathrm{I}) \times(\mathrm{N}+\mathrm{I})} \\
& \mathbf{K}_{\mathrm{Vu}}^{*} \in \mathbb{R}^{(\mathrm{N}+\mathrm{I}) \times(\mathrm{N}+\mathrm{I})} \\
& \boldsymbol{F}^{*} \in \mathbb{R}^{(\mathrm{N}+\mathrm{I})} \\
& \mathbf{Q}^{*} \in \mathbb{R}^{(\mathrm{N}+\mathrm{I})} \\
& \mathbf{u}^{*}, \boldsymbol{V}^{*} \in \mathbb{R}^{(\mathrm{N}+\mathrm{I})} \\
& \mathbf{u}, \quad v \in \mathbb{R}^{N_{u}}, \mathbb{R}^{N_{v}}
\end{aligned}
$$

Coupled reduction with distributed electric parameters (CD). Our method for modal truncation of piezoelectrically coupled systems differs from the commonly used method with lumped electric parameters and mechanical mode shapes in three important aspects: (1) In contrast to the approach, described in section "Mechanical reduction with lumped electric parameters (ML)," we do not reduce the electric part to a lumped parameter, but rather take the local potential distribution into account. (2) Moreover, we solve here the generalized eigenvalue problem for the coupled system (equation (8)) for a number of $N$ eigenvectors (equation (15)), thereby considering coupling already at the stage of mode shape computation. (3) By introducing a socalled static mode shape, mechanical deflection is also computed correctly when the structure is excited electrically. Furthermore, the influence of mechanical boundary conditions on the quasistatic electric capacitance is captured. We will now describe the approach and assess its differences to the traditional coupling approach in section "Evaluation of model performance." The mathematical procedure is summarized in Table 1.

The generalized eigenvalue problem (equation (15)) of the full coupled system (equation (8)) is solved for a number of $N$ eigenmodes that are arranged columnwise in the matrix $\boldsymbol{\Phi}$. The column vector $\phi_{\mathrm{p}}$ is the solution to a static problem under electric excitation (equation (16)). The result of this calculation is a static deflection curve as well as its corresponding electric potentials that generally differ from the dynamic mode shapes of the free-vibration problem. In the following, it is denoted by static mode, as we treat the deflection curve like a mode shape in the reduction process. The transformation matrices $\boldsymbol{\Phi}_{\mathrm{u}}$ and 
$\boldsymbol{\Phi}_{\mathrm{V}}$ (equation (17)) contain the mechanical and electric parts of the eigenvectors from equation (15) as well as the solution to equation (16), respectively. Note, that the electric parts of the eigenvectors are not the eigenvectors of the electric stiffness matrix, but the electric nodal potentials, corresponding to a mechanical mode shape with piezoelectric coupling. Here, $N$ is the number of eigenvectors that are computed in equation (15). $N_{\mathrm{u}}$ and $N_{\mathrm{V}}$ indicate the number of mechanical and electric degrees of freedom from the original FE problem (equation (8)), respectively. The mechanical and electric FE matrices and the right-hand-sides are reduced separately with the help of the respective matrices of eigenvectors (equation (18)). The reduced versions are marked by a superscript asterisk. The reduced mechanical, electric, and coupling matrices are subsequently re-assembled into the structure of equation (8) for harmonic calculations or inserted into the Simulink structure of Figure 1 for transient simulations. The solution to the reduced problem is given in generalized coordinates. In order to extract nodal displacements or potentials, the reduced solution vector $\boldsymbol{V}^{*}$ or $\boldsymbol{u}^{*}$ has to be multiplied by the respective transformation matrix (equation (19)). The starting point of the procedure are the three FE matrices and the right-hand-side vector in equation (8), which are obtained from the FE software. All computations are subsequently performed in MATLAB. As all workspace variables are available in Simulink, no further interfacing has to be implemented.

Diagonalization and damping model. Due to our modifications to the reduction method, the orthogonality with respect to the FE matrices is lost. We have not experienced any numerical issues, related to the loss of orthogonality. Diagonal system matrices exhibit, however, a very useful property regarding the damping model. When the ODEs are decoupled, a specific damping factor can be assigned to each mode. Damping can, therefore, be modeled as constant over the whole frequency range of interest or as frequency-dependent obeying an appropriate damping model. When the matrices are not diagonal, it is common to use a Rayleigh damping model (Kaltenbacher, 2015). Here, a damping matrix is assembled as a superposition of the weighted mass matrix and stiffness matrix. With this model, a damping matrix can only represent a given damping factor at one discrete frequency. In harmonic simulations, the damping matrix can easily be adjusted at each frequency step to follow a given frequency-dependent or independent damping behavior. When simulating in the timedomain, this is not possible, and damping can only be prescribed at one discrete frequency component.

To overcome this shortcoming and additionally gain simulation efficiency, the reduced matrices (equation (18)) can be diagonalized. Thereby, the reduced matrices are re-assembled into the structure of the original FE system (equation (8)). A generalized eigenvalue

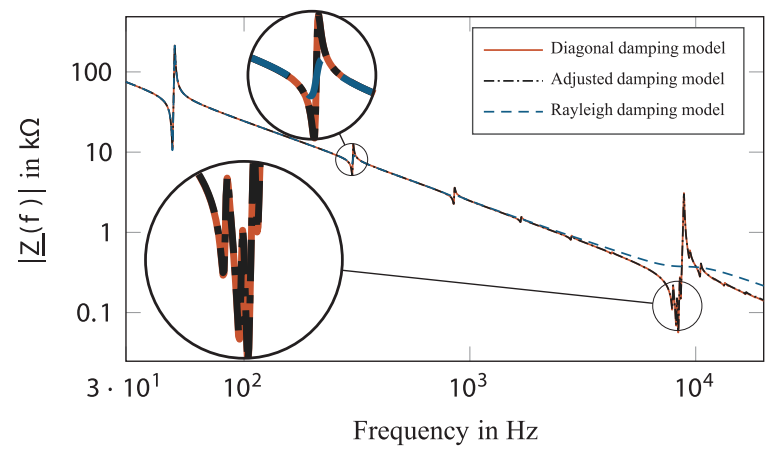

Figure 3. Electric impedance of the unimorph structure from Figure 4 using three different damping models.

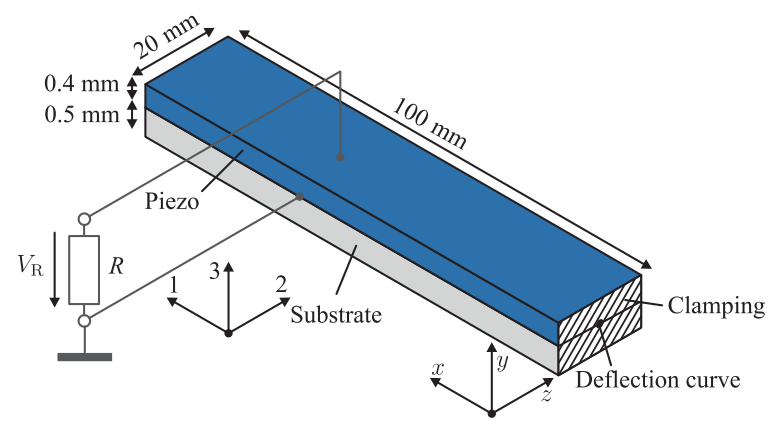

Figure 4. Unimorph cantilever consisting of passive substrate and a piezoelectric layer. The hatched area marks the mechanical clamping in all directions. The piezo material is covered by electrodes on its top and bottom area.

problem of the reduced system is solved, this time without truncating the eigenvectors. The resulting eigenvectors are used for a diagonalization of the reduced matrices. Subsequently, a distinct damping factor can be assigned to each equation on the diagonal of the damping matrix. Due to diagonalization, the ODEs are decoupled, each representing one single modal component. In Figure 3, we compare the electric impedance of the unimorph cantilever from Figure 4 for three different damping models, namely (1) Rayleigh model, (2) Rayleigh model with adjusted damping, and (3) diagonalized damping matrix. The Rayleigh model reproduces a prescribed damping factor at a frequency of $47 \mathrm{~Hz}$ and exhibits increasing effective damping toward higher frequencies. For the adjusted damping model, a new damping matrix has been assembled for each frequency using the same damping factor. The curve for the diagonal damping model was calculated in a transient Simulink simulation with subsequent Fast fourier transform (FFT) using a chirp signal as input.

The diagonal damping model offers the same damping behavior as the adjusted damping model, although the damping matrix is not adjusted during the calculation. In transient simulations with broadband excitation, a model with Rayleigh damping, on the other 


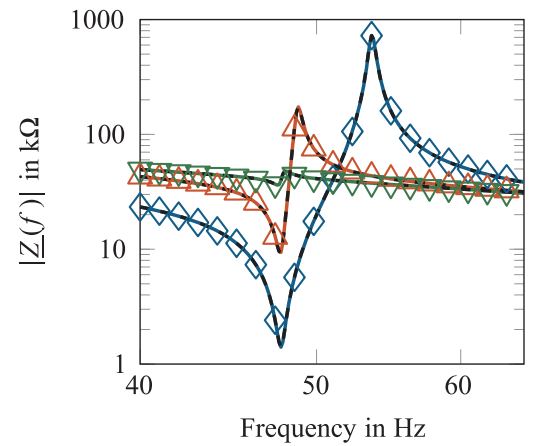

(a)

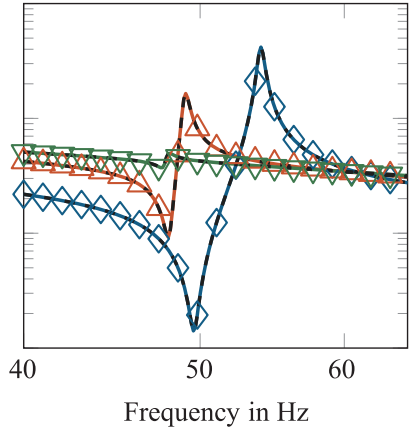

(b)

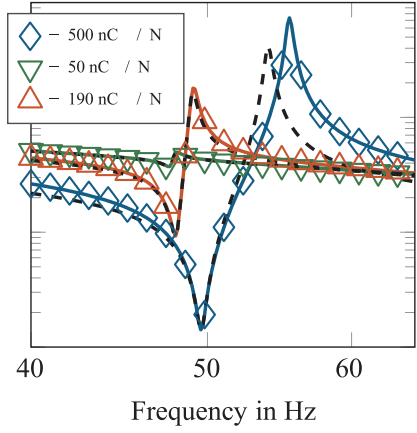

(c)

Figure 5. Electric impedance of the unimorph cantilever (cf. Figure 4) with different transverse coefficients $d_{31}$ : (a) lumped parameter approach (ML). Model derived from 2D FE model (solid) and Erturk model (dashed); (b) full FE model (dashed), CD model (solid); (c) full FE model (dashed), hybrid model with distributed parameters and mechanical mode shapes (solid).

hand, is not able to represent prescribed damping for more than one modal component. Figure 3 reveals that damping is significantly increased already for the second bending mode. The resonances around $9 \mathrm{kHz}$ are almost eliminated from the model by the high damping.

\section{Evaluation of model performance}

In this section, the main differences between our modified approach and traditional reduced modeling of piezoelectric structures are examined. We will use the unimorph cantilever structure, published in Erturk and Inman (2008a) with the same dimensions and material parameters, if not indicated otherwise. We will investigate the effect of distributed electric parameters in the following section as well as the effect of coupled mode shapes and the static mode shape in section "Influence of coupled mode shapes."

\section{Effect of distributed electric parameters}

One of the main advantages of our reduction approach is the absence of simplification in electrical behavior by lumped parameters. Introducing a lumped capacitance implies the assumption of a homogeneous and constant electric field distribution. Whenever an electromechanical structure is strongly coupled, however, the field distribution is being influenced by the mechanical mode shape and vice-versa.

In Figure 5, we compare the electric impedance of the unimorph cantilever from Figure 4 for different transverse piezoelectric coefficients $d_{31}$. The parameter value $d_{31}=-190 \mathrm{nC} / \mathrm{N}$ is the original value from Erturk and Inman (2008a) as are all other material parameters. Figure 5(b) shows our reduced model (CD) together with the solution to the full FE model. Increasing the $d_{31}$ parameter causes an increase of both the resonance frequency as well as the anti-resonance frequency. In Figure 5(a), we used the Erturk model from Erturk and Inman (2008a), which produces the same results as the mechanical reduction approach (ML) from Figure 2. It can be observed that the resonance frequency is not affected by the $d_{31}$ parameter in an electrically lumped model. In Figure 5(c), we introduced a hybrid form of model, where the distributed potentials are being considered, but the mechanical mode shapes have been computed without piezoelectric coupling. Here, the resonance frequency behaves as in the full FE model with respect to the $d_{31}$ parameter. The difference in magnitude between anti-resonance and resonance, however, is overestimated, compared to the $\mathrm{CD}$ model. The same overestimation occurs in the ML approach (cf. Figure 5(a)). We will refer to this shortcoming in section "Influence of coupled mode shapes." With a view to justifying the importance of distributed potential, let us take a closer look at the equipotential lines in Figure 6, which were reconstructed from our reduced model (CD) for the three different piezoelectric coefficients at the corresponding resonance frequency. While for the small coefficient of $d_{31}=-50 \mathrm{nC} / \mathrm{N}$, the potential distribution is very similar to that in a plate capacitor, it considerably deviates from such a homogeneous field for increasing $d_{31}$. Hence, introducing a lumped parameter for the description of the electric field does not correctly reflect the physical field, involved in the coupling. The systematic modeling error is negligible for weak coupling or for very thin piezoelectric layers but becomes problematic whenever the real distribution differs significantly from that of a homogeneous electric field. It should also be noted that the field distribution is frequency dependent, as the mechanical mode shapes change with excitation frequency.

Figure 7 shows a $d_{33}$ mode bending cantilever. Here, segments of oppositely poled layers of piezoelectric material are interconnected in parallel (e.g. PI Power Patch (PI (Ceramic GmbH, 2016)). For this kind of setup, the effect of non-homogeneous potential distribution can be 


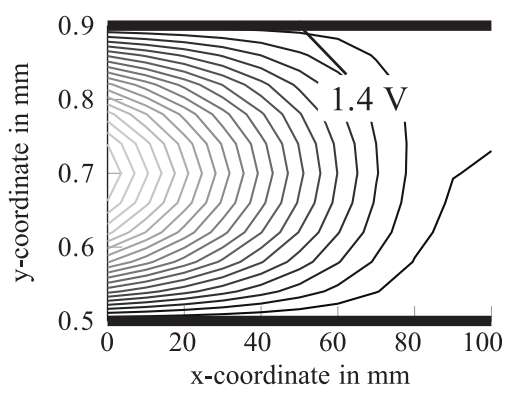

(a)

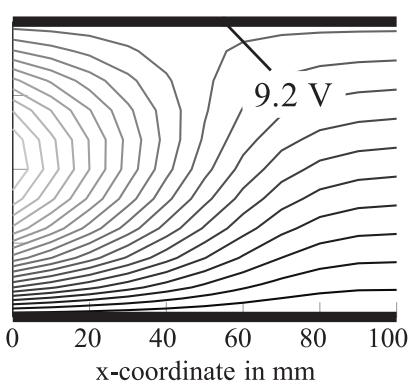

(b)

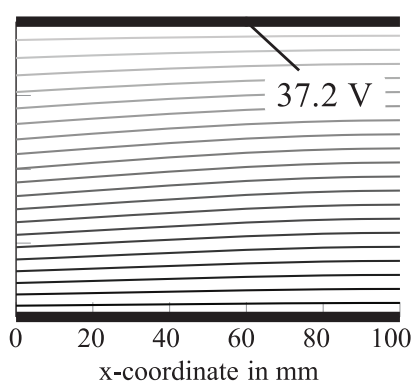

(c)

Figure 6. Equipotential lines within the piezoelectric material in Figure 4 from our CD model at respective resonance frequency (Figure 5). Excitation was an electric current with amplitude I $\mathrm{mA}$ : (a) $d_{31}=-500 \mathrm{nC} / \mathrm{N}$, (b) $d_{31}=-190 \mathrm{nC} / \mathrm{N}$, and (c) $d_{31}=$ $-50 \mathrm{nC} / \mathrm{N}$.

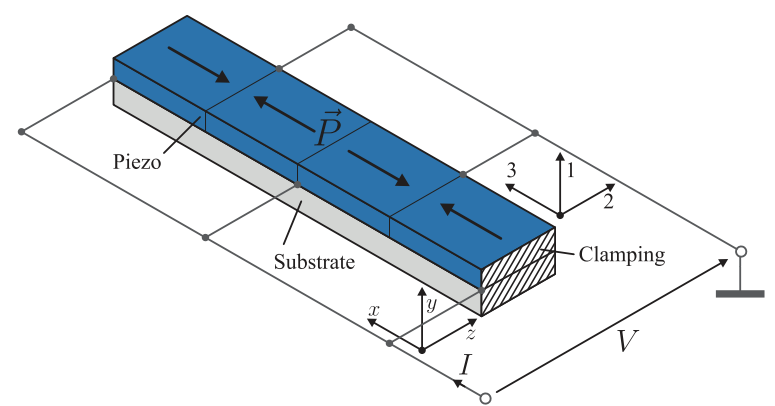

Figure 7. Unimorph cantilever with $d_{33}$ mode piezoelectric material. The segments are oppositely poled and interconnected in parallel. Dimensions are equal to Figure 4.

observed especially at the second bending mode vibration. Figure 8(a) shows the electric impedance for a piezoelectric layer divided into 2 and into 50 segments, respectively. The curve for the two-segment model has been corrected by factor $25^{2}$ to account for its lower electrostatic capacitance. The 50 -segment layer has 25 times as many layers connected in parallel, which are by a factor of 25 thinner, compared to the two-segment cantilever. Although the mechanical properties of both setups are identical, the second bending mode resonance frequency is much higher for the two-segment setup. In Figure 8(b), the lumped modeling approach shows no alteration in resonance frequency between the two geometries. Again, the hybrid model with distributed potentials and purely mechanical mode shapes is shown for comparison. From Figure 9(b), it becomes clear that the distributed potentials have a significant effect on the effective stiffness of the structure, when only two segments, that is, one artificial equipotential area is present in the material. Figure 9(a) shows two segments from the 50 -segment cantilever. Due to the much thinner piezoelectric layers, the displacement gradient over one single layer is almost constant, which leads to a nearly homogeneous field intensity. With those boundary conditions, the lumped modeling approach is appropriate and the

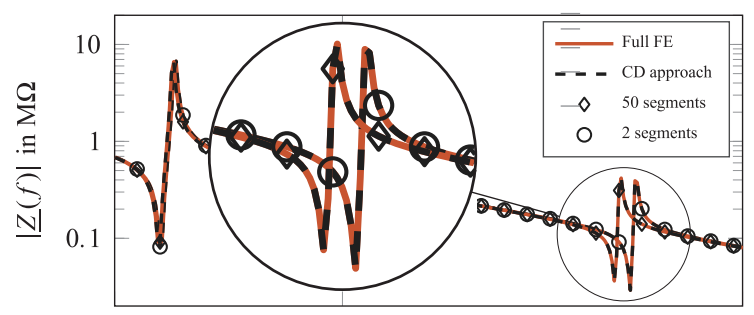

(a)

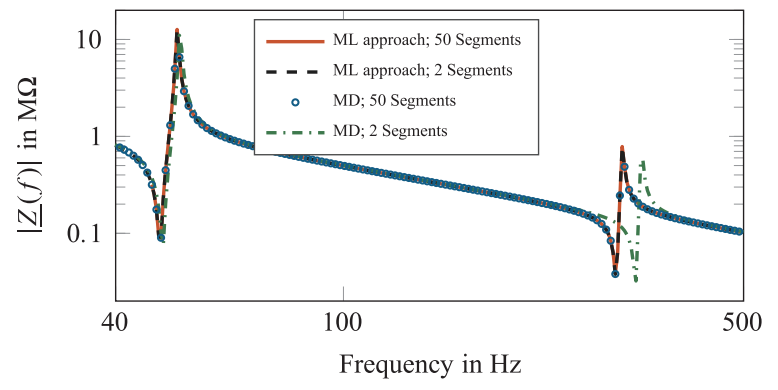

(b)

Figure 8. Electric impedance of the $d_{33}$ mode harvester (Figure 7): (a) CD model and full FE model for 2 and 50 segments and (b) comparison of ML model and hybrid model with mechanical mode shapes and distributed electric parameters (MD). Curves for two segments have been multiplied by $25^{-2}$.

two different modeling approaches produce almost identical results.

\section{Influence of coupled mode shapes}

The second important difference between our reduction method (CD) and traditional approaches (ML) is the consideration of coupling already at the stage of computing the mechanical mode shapes. When solving the generalized eigenvalue problem (equation (15)), piezoelectric coupling has an influence on the mechanical displacements, depending on material parameters and geometry. Another important aspect is our static mode 


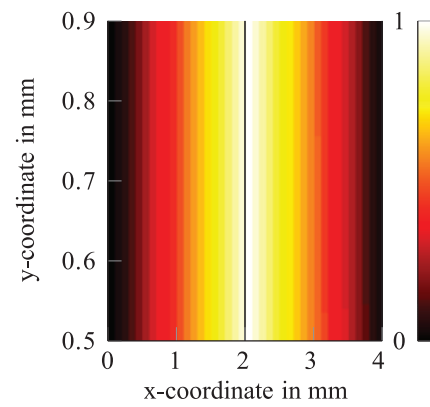

(a)

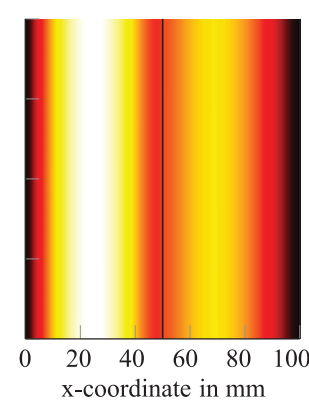

(b)
Figure 9. Normalized local potential distribution in the piezoelectric material of the $d_{33}$ mode harvester (Figure 7) at respective second resonance frequency: (a) two segments out of 50 and (b) two segments out of 2 .

shape (equation (16)) that affects mechanical behavior under electric excitation. It also captures the influence of piezoelectric coupling and mechanical boundary conditions on the quasistatic capacitance and, therefore, also on the effective coupling factors.

In Figure 10(b), lines of displacements of the model from Figure 4 are shown under acceleration in opencircuit condition. We applied an acceleration in $\mathrm{y}$-direction at a frequency of $200 \mathrm{~Hz}$ and, after backtransformation of the results (equation (19)), extracted the displacements of a line in $\mathrm{x}$-direction at $y=0.5 \mathrm{~mm}, z=10 \mathrm{~mm}$ (denoted by "deflection curve" in Figure 4). It can be observed that the shape of the forced vibration coincides for the reduced and the full FE model. For the modal truncation, we used the first two eigenvectors, which are shown in Figure 10(a), and the additional static mode shape in a second simulation. For the electric excitation of the cantilever, we applied an electric charge at $200 \mathrm{~Hz}$ on the top electrode. As can be seen in Figure 10(c), the vibration shape is not correctly reproduced by the reduced system of equations if only the first two eigenvectors are considered. However, if the static mode is added as an additional column vector $\phi_{\mathrm{p}}$ to the transformation matrix (equation (17)), the deflection curve under electric charge excitation is correctly represented by the reduced system of equations. The same holds, when we study the electric voltage over a connected electric load under acceleration as well as the electric field inside the piezoelectric material (cf. Figure 6).

Furthermore, the static mode leads to a correctly reproduced quasistatic capacitance value under different mechanical boundary conditions. In the following, let us investigate the behavior of the different modeling approaches under varying boundary conditions and material parameters. Mechanical boundary conditions play an important role for the electrically measured quantities in piezoelectric materials. In our FE formulation, we use the stress-charge form (i.e. e-form) of the piezoelectric constitutive equations (4) - (5). The tensor of dielectric permittivity $\left[\varepsilon^{S}\right]$ is the permittivity of the material at constant strain, that is, when the specimen is completely clamped. The piezoelectric effect has, therefore, no influence on the measured capacitance. In contrast, in the strain-charge form (i.e. d-form) (equation (20)), the tensor of permittivity $\left[\boldsymbol{\varepsilon}^{T}\right]$ is given at constant stress, that is, in unclamped conditions. When characterizing material parameters, this is usually done for a simplified geometry in strain-charge form and measurements are carried out in unclamped conditions (Institute of Electrical and Electronics Engineers (IEEE), 1988). The constitutive equations can then be transformed into each other as they are mathematically equivalent. To obtain the permittivity at constant strain, the piezoelectric effect on the apparent permittivity has to be subtracted (equation (21))

$$
\begin{gathered}
D=[\boldsymbol{d}] \boldsymbol{\sigma}+\left[\boldsymbol{\varepsilon}^{T}\right] \boldsymbol{E} \\
{\left[\boldsymbol{\varepsilon}^{S}\right]=\left[\boldsymbol{\varepsilon}^{T}\right]-[\boldsymbol{d}]\left[\boldsymbol{c}^{\boldsymbol{E}}\right][\boldsymbol{d}]^{\mathrm{T}}}
\end{gathered}
$$

An electromechanical model should reflect this effect of mechanical boundary conditions on electric behavior, regardless of which form of constitutive equation

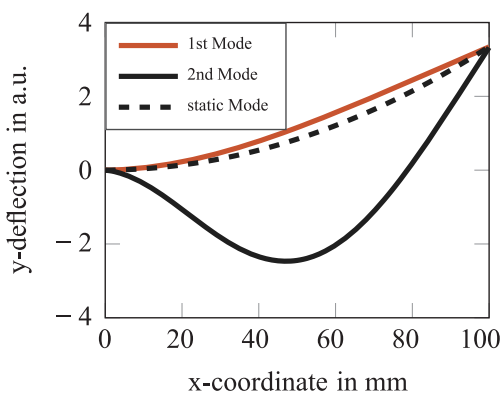

(a)

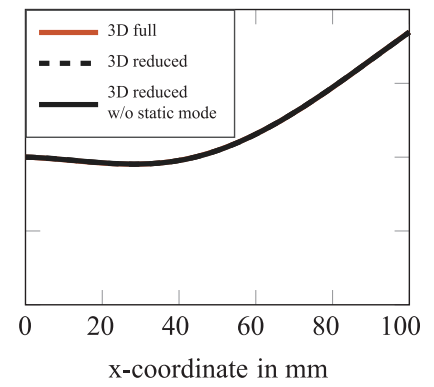

(b)

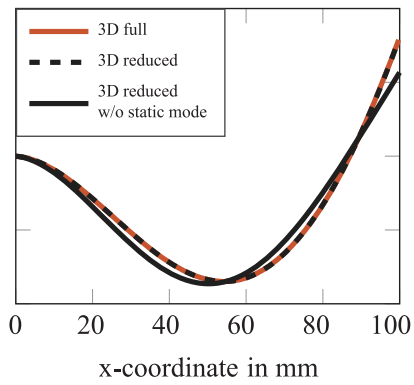

(c)

Figure 10. Deflection curves of the model in Figure 4 under different excitations in arbitrary units (a.u.): (a) first two eigenmodes of the free-vibration problem and static deflection curve under electric excitation, (b) deflection curves under mechanical acceleration at $200 \mathrm{~Hz}$, and (c) deflection curves under electric excitation at $200 \mathrm{~Hz}$. 


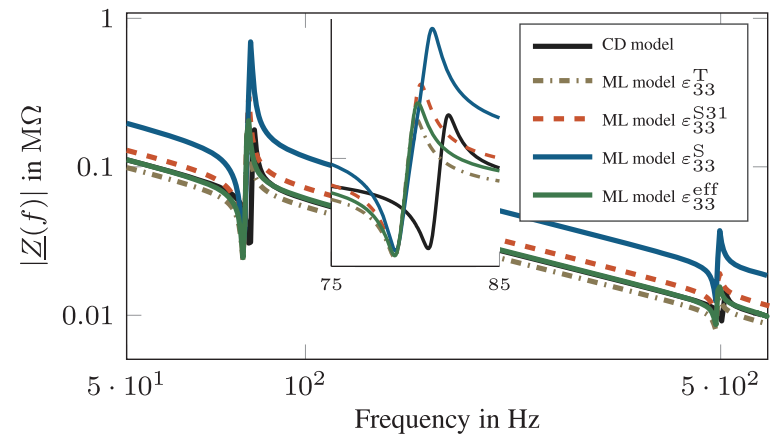

Figure I I. Electrical impedance of the unimorph cantilever (Figure 4) for $h_{\mathrm{p}}=1 \mathrm{~mm}$. The traditional model was computed for different dielectric permittivities, including $\varepsilon^{\text {eff }}$ from Figure 12.

has been used in its deduction. With a view to investigating the effect of mechanical boundary conditions on electrical quantities, we use a full set of transversalisotropic material data, typical for PZT-5A (equations (23) to (25)) (Appendix 1) with a 3D model of the unimorph cantilever from Figure 4. Figure 11 displays the electrical impedance from our modified approach (CD), together with a number of curves from the ML model that does not consider coupling at the stage of mode shape calculation. We used different permittivities, ranging from ideally unclamped $\left[\boldsymbol{\varepsilon}^{T}\right]$ to ideally clamped material $\left[\boldsymbol{\varepsilon}^{\boldsymbol{S}}\right]$. The parameter $\left[\boldsymbol{\varepsilon}^{\boldsymbol{S 3 1}}\right]$ is obtained, when only $d_{31}$ is used in equation (21). This would assume that the material behaves as clamped in 1-direction and free in 3-direction. As can be observed from the impedance curves, neither of the clamped or unclamped parameters reflect the mechanical boundary conditions at hand. In order to assess the error, introduced by this systematic modeling flaw, a Nelder-Mead algorithm was used (Nelder and Mead, 1965) to search for an effective permittivity $\left[\varepsilon^{\text {eff }}\right]$ that would give rise to the same quasistatic capacitance in both models under the given boundary conditions.

Furthermore, we varied the thickness $h_{\mathrm{p}}$ of the piezoelectric layer between $1 \times 10^{-5} \mathrm{~mm}$ and $1 \mathrm{~mm}$, computing the effective permittivity $\left[\boldsymbol{\varepsilon}^{\text {eff }}\right]$ for each thickness. The result is plotted in Figure 12, along with the three aforementioned permittivities under ideal boundary conditions. The variation in thickness represents, in effect, a gradual change in boundary conditions for the piezoelectric layer. As the thickness approaches zero, the film is effectively clamped in 1-direction and partially clamped in 3-direction. The effective permittivity is closer to $\left[\boldsymbol{\varepsilon}^{S}\right]$ than to $\left[\boldsymbol{\varepsilon}^{T}\right]$. For thick layers of piezoelectric material, its movement is barely hindered by the substrate in 1-direction and practically free in 3direction. The value of $\left[\boldsymbol{\varepsilon}^{\text {eff }}\right]$ approaches $\left[\varepsilon^{T}\right]$ asymptotically. When simplifying the material properties such that only the $d_{31}$ effect is considered, the material is

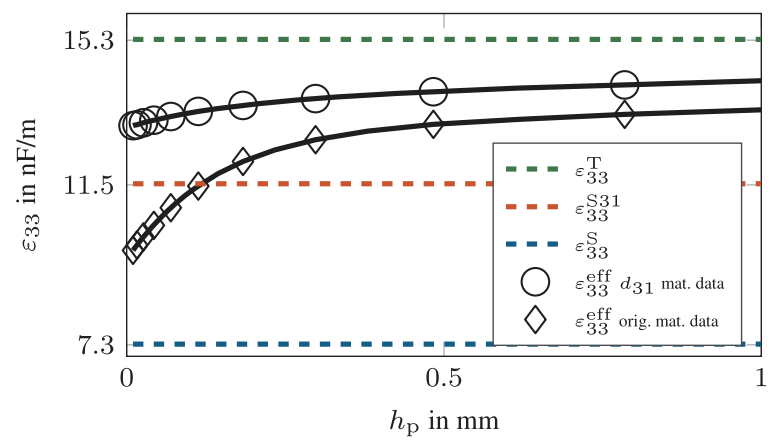

Figure I2. Effective dielectric permittivity over thickness of the piezoelectric layer $h_{\mathrm{p}} . \varepsilon_{\text {eff }}$ is the permittivity of the ML model that yields the same quasistatic capacitance as the full FE model it was derived from.

implicitly considered free in three-direction. This leads to $\left[\boldsymbol{\varepsilon}^{\text {eff }}\right]$ taking a higher value, in the range between $\left[\boldsymbol{\varepsilon}^{\mathbf{S 3 1}}\right]$ and $\left[\boldsymbol{\varepsilon}^{T}\right]$. We chose here to show the effect of quasistatic coupling with the help of the unimorph example at hand. The effects are, however, more drastic, if clamping conditions are altered in the course of finding an optimal design, for example, deviating from the idealized clamped-free cantilever.

The preceding analysis has been focused on the effect of quasistatic coupling on the apparent capacitance of the structure, that is, a constant factor in the impedance plot. Neglecting piezoelectric coupling at the stage of modal analysis of the full model can also lead to erroneous mode shapes. Physically, the piezoelectric effect stiffens the material because the shift in center of electric charge, emerging from mechanical deformation, leads to additional mechanical stress. Therefore, the mode shape will generally differ from a purely mechanical mode shape. The difference in strain, in case of a clamped-free unimorph cantilever, is negligible in 1-direction due to the strong mechanical coupling between piezoelectric material and substrate. For the 3-direction, however, a significant discrepancy can be observed from Figure 13. The plot shows the error for the $y y$-component of the strain normalized to the absolute maximum strain at the first bending eigenmode. The mechanical strain is overestimated (locally) by almost $10 \%$ when coupling is not considered in the reduction process. This can lead to overestimation of coupling parameters and effective coupling factors.

As both, coupling parameters and quasistatic capacitance have a strong influence on electromechanical coupling, we compared our modified modeling approach (CD) to the traditional one (ML), when attempting a geometrical optimization of the unimorph cantilever. To this end, we performed a parameter study over the thickness of the piezoelectric layer, computing the effective coupling factor $k_{\mathrm{eff}}^{2}$ at the first resonance-antiresonance couple 


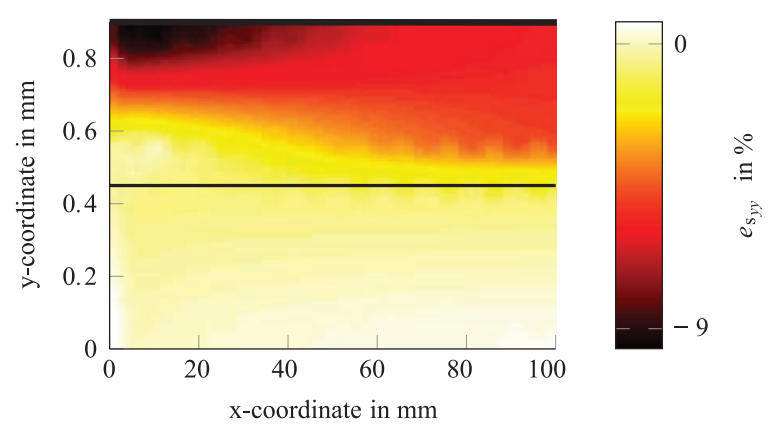

Figure 13. Error in the strain component $s_{y y}$, normalized to the absolute maximum strain at the respective first bending mode.

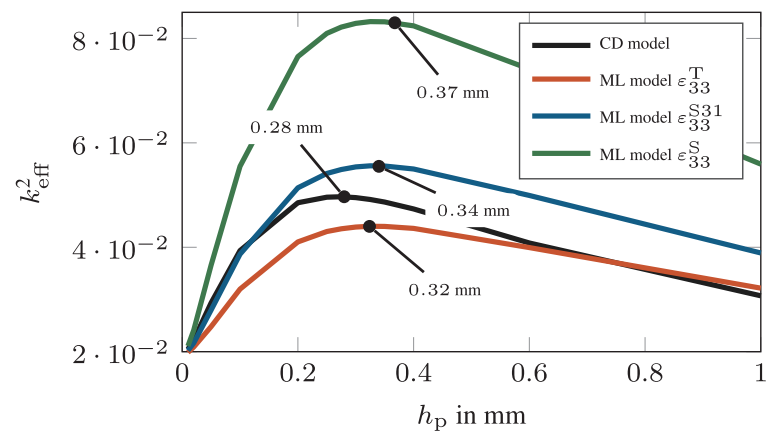

Figure 14. Effective coupling factor at the first bending eigenfrequency over thickness of the piezoelectric layer $h_{p}$. Coupling was computed for three different dielectric permittivities for the ML model. Maxima are indicated with their corresponding optimal thickness.

$$
k_{\mathrm{eff}}^{2}=\frac{f_{\mathrm{ar}}^{2}-f_{\mathrm{r}}^{2}}{f_{\mathrm{r}}^{2}}
$$

Indices "ar" and " $\mathrm{r}$ " stand for anti-resonance and resonance, respectively. In Figure 14, the result of effective coupling over piezo thickness is shown for our CD approach and three different permittivity parameters with the ML approach. The indicated optimal thicknesses where again found using a Nelder-Mead optimization algorithm. As suggested earlier, electromechanical coupling differs significantly between the two models. Equally importantly, in case of using mechanical mode shapes, the result is strongly dependent on the choice of permittivity parameters, which for the ML model cannot reliably be determined using the standard procedure (IEEE, 1988) or an inverse method (Rupitsch and Ilg, 2015; Rupitsch and Lerch, 2009).

In summary, our newly introduced modeling components - the distributed electrical parameters, as well as the piezoelectrically coupled mode shapes and the static mode shape - close the gap toward physically consistent reduced models. While simplification, in some cases, may introduce only negligible errors, it renders the models physically inconsistent with the basic

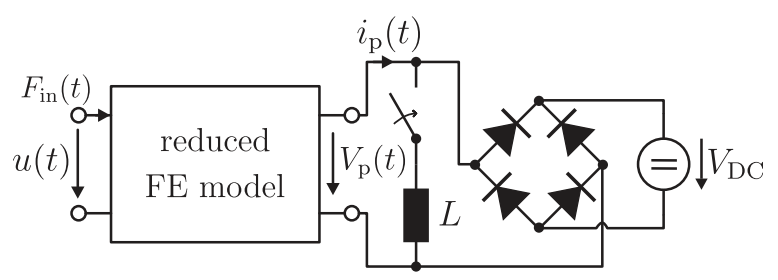

Figure 15. Circuit of the SSHI technique connected to the reduced FE model of the piezoelectric VEH (cf. Figure I).

equations they were originally derived from. This constitutes a systematic error in modeling methodology that should be avoided.

\section{Application example}

With a view to showing the applicability of our approach, let us discuss an example that is concerned with a power electronic circuit, connected to a VEH. It demonstrates the feasibility of our approach in transient system simulations of VEHs. The SSHI (synchronized switch harvesting on inductor) circuit is a means of extracting electrical energy from a vibrating piezoelectric energy harvester (Figure 15). The working principle relies on a resonant circuit consisting of the capacitance of the piezoelectric material and an external inductor $L$. The inductor is connected in parallel through a switch to the harvester's electrode. When the open-circuit voltage reaches a maximum, the switch is closed, which results in an electrical oscillation. During the zero-crossing of the inductor current, the switch is opened and the voltage amounts to the inverted value (neglecting resistive losses). As the electrical oscillation frequency is usually by orders of magnitude higher than the mechanical excitation frequency, the inversion of the voltage is almost instantaneous. Behind the inductor stage, a diode rectifier connects the harvester to a capacitor or a battery for energy storage. We assumed a constant voltage $V_{\mathrm{DC}}$ to emulate an ideal energy storage device. Through the rapid inversion of the voltage, the technique aims at extending the time fraction during which the diodes are conducting. Therefore, in certain applications, more energy can be extracted from the harvester, compared to a diode rectifier. For details about this technique, we would like to refer to the literature (e.g. Deterre et al., 2012; Lefeuvre et al., 2009; Shu et al., 2007; Yiming et al., 2008).

As piezoelectric energy harvester, we chose the unimorph cantilever example from Figure 4 (3D FE model) with the material parameters from equations (23) to (25). The harvester was excited by an acceleration of $a_{\text {in }}(t)=$ $1 \mathrm{~m} / \mathrm{s}^{2} \sin (2 \pi t 48.7 \mathrm{~Hz})$ in y-direction. The DC voltage was $V_{\mathrm{DC}}=1.8 \mathrm{~V}$ and the inductivity $L=0.1 \mathrm{mH}$. The diodes were modeled by a drop voltage of $V_{\text {drop }}=0.6 \mathrm{~V}$ and a resistance of $0.3 \Omega$ in forward direction. 


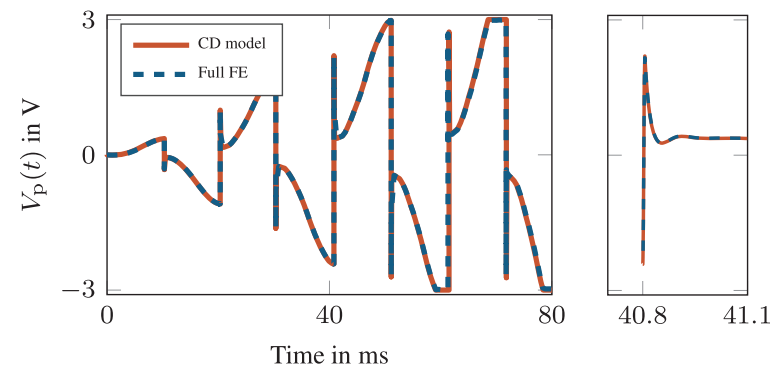

Figure 16. Time response of the voltage $V_{p}(t)$ from Figure 15 . The magnified portion reveals the equivalence of the reduced model and the full FE model with 15 eigenvectors involved in the reduction.

With a view to validating our reduction approach, we performed simulations with different numbers of eigenvectors (EVs) in the reduction process. Although the excitation frequency is in the range of the first eigenfrequency of the harvester, it is not sufficient to consider only one or two eigenmodes. Figure 16 shows the solution of a transient simulation considering 15 eigenvectors. The need for a larger number of eigenmodes originates from the short pulses of electric current that is drawn from the piezoelectric material during the voltage inversion. Their broadband spectrum has significant effects on the vibration of the harvester. It should be noted that we performed the same experiment with the truncated model with mechanical eigenmodes. Significant differences in $V_{\mathrm{p}}(t)$ could be observed.

A direct validation as in the case of the timeharmonic simulations is not possible here, because the full FE model cannot be computed in Simulink due to restrictions on the size of the input data (the threedimensional model has 76,188 degrees of freedom). Instead, we pursued an indirect validation approach visualized in Figure 17. After a number of consecutive time-domain simulations of the reduced model with increasing numbers $N$ of eigenmodes, the solutions for the piezoelectric voltage and current converged for $N=15$. Convergence was established by visual inspection of the results. We stored the piezoelectric charge $q_{\mathrm{p}}(t)=\int_{0}^{t} i_{\mathrm{p}}(\tau) \mathrm{d} \tau$ from the Simulink simulation with 15 eigenmodes and supplied it, along with the mechanical acceleration, to the FE solver CFS++. A forward simulation of the full FE model was conducted with the same mechanical excitation and the charge input, emerging from the algebraic loop of the coupled Simulink model. We claim that the reduced solution for $N=15$ is accurate, because the voltage $V_{\mathrm{p}}(t)$ from the full FE simulation matches the solution to the reduced model (cf. Figure 16). The calculation of 15 eigenmodes accounted for $320 \mathrm{~s}$ of computation time. In the timedomain simulation, a time step required $2.6 \mathrm{~ms}$ on average against $2 \mathrm{~s}$ for the full $\mathrm{FE}$ simulation in $\mathrm{CFS}++$. It should also be noted that the fast oscillation during the

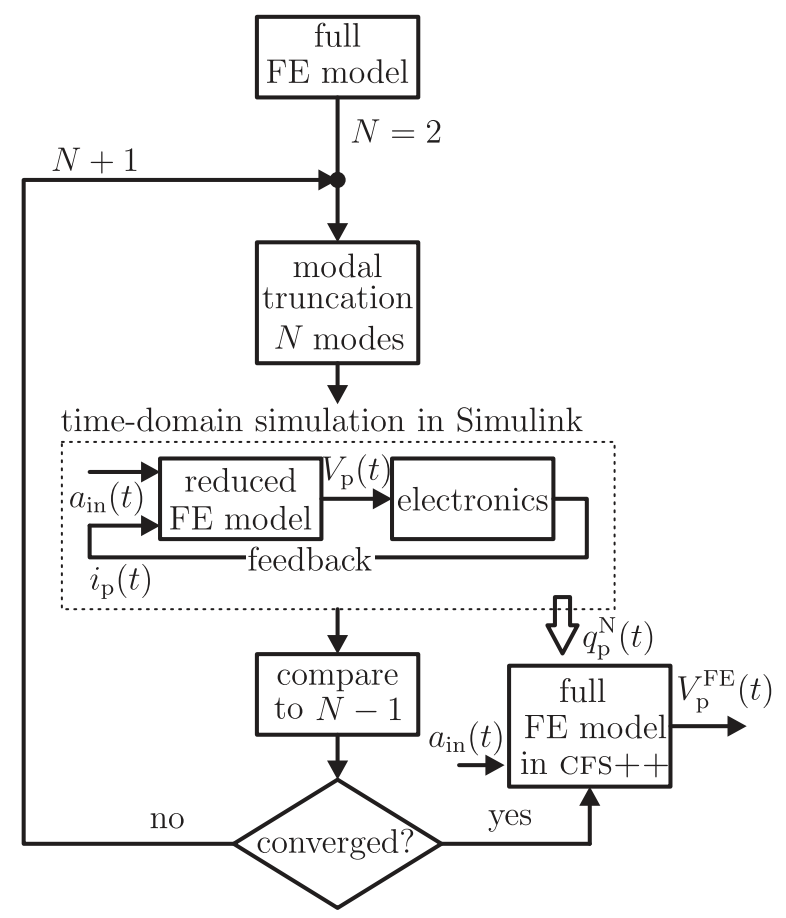

Figure I7. Flowchart of the validation procedure for timedomain simulations in example $\mathrm{I}$.

switching process requires a significant refinement of the step size compared to the rest of the simulation. Thus, a variable-time-step solver is essential for a time efficient computation. In the forward simulation of the full FE system, a fixed time step of $1 \mu$ was used leading to $80 \times 10^{3}$ time steps. This required over $30 \mathrm{~h}$ of simulation time. The implicit variable-step-solver, which is used in the Simulink simulation results in less than $8 \times 10^{3}$ time steps and, in conjunction with the reduced order of the model, in an overall simulation time in the seconds regime.

\section{Conclusion}

An iteratively coupled scheme was described, which allows hybrid simulations of piezoelectric FE models and electric circuits. The MATLAB/Simulink environment is exploited for its convenient capabilities of handling both ordinary differential equations and network representations of electric circuits. In order to perform hybrid simulations efficiently, we introduced a modified distributed parameter modal truncation procedure for the piezoelectric FE formulation with direct coupling. The reduced model was validated against full FE models in the frequency-domain and in the timedomain by means of different examples. Furthermore, we buttressed the advantages of our modifications over existing truncation methods by identifying important shortcomings of traditional methods with regard to the underlying physics of piezoelectric coupling. By applying the coupling scheme in combination with the modal 
truncation technique, energy harvesters can be simulated in conjunction with power electronic circuits. Due to the accurate reproduction of the full FE models, geometrical variations can be accounted for within the possibilities of the FEM. The need for an appropriate number of eigenmodes to be considered for the reduction of a specific problem was pointed out. It is possible to reconstruct important results, like material stresses or electric field distribution, from the solution to the reduced model without significant loss of accuracy. An assessment of the computational cost of full and reduced representations revealed the efficiency of the proposed order reduction technique.

\section{Declaration of conflicting interests}

The author(s) declared no potential conflicts of interest with respect to the research, authorship, and/or publication of this article.

\section{Funding}

The author(s) disclosed receipt of the following financial support for the research, authorship, and/or publication of this article: This research was supported by the Deutsche Forschungsgemeinschaft (DFG) in context of the Collaborative Research Centre/Transregio 39 PT-PIESA (subproject (06) and the Bavarian Ministry of Economic Affairs and Media, Energy and Technology as part of the Bavarian project "Leistungszentrum Elektroniksysteme (LZE)."

\section{References}

Bathe KJ (2006) Finite Element Procedures. Klaus-Jürgen Bathe.

Beeby SP, Wang L, Zhu D, et al. (2013) A comparison of power output from linear and nonlinear kinetic energy harvesters using real vibration data. Smart Materials and Structures 22(7): 075022.

Challa VR, Prasad MG, Shi Y, et al. (2008) A vibration energy harvesting device with bidirectional resonance frequency tunability. Smart Materials and Structures 17(1): 015035.

Daniels A, Zhu M and Tiwari A (2013) Evaluation of piezoelectric material properties for a higher power output from energy harvesters with insight into material selection using a coupled piezoelectric-circuit-finite element method. IEEE Transactions on Ultrasonics, Ferroelectrics, and Frequency Control 60(12): 2626-2633.

Deterre M, Lefeuvre E and Dufour-Gergam E (2012) An active piezoelectric energy extraction method for pressure energy harvesting. Smart Materials and Structures 21(8): 085004.

Elvin NG and Elvin AA (2009a) A coupled finite element circuit simulation model for analyzing piezoelectric energy generators. Journal of Intelligent Material Systems and Structures 20(5): 587-595.

Elvin NG and Elvin AA (2009b) A general equivalent circuit model for piezoelectric generators. Journal of Intelligent Material Systems and Structures 20(1): 3-9.
Erturk A and Inman DJ (2008a) A distributed parameter electromechanical model for cantilevered piezoelectric energy harvesters. Journal of Vibration and Acoustics 130(4): 041002.

Erturk A and Inman DJ (2008b) Issues in mathematical modeling of piezoelectric energy harvesters. Smart Materials and Structures 17(6): 065016.

Gedeon D, Rupitsch SJ and Lerch R (2014) Numerical and experimental study on the frequency tuning characteristics of a rotating piezoelectric energy harvester. In: Ultrasonics symposium (IUS), 2014 IEEE international, Chicago, IL, 3-6 September, pp. 2486-2489. Chicago, IL: IEEE.

Gu L and Livermore C (2012) Compact passively self-tuning energy harvesting for rotating applications. Smart Materials and Structures 21(1): 015002.

Hendijanizadeh M, Sharkh SM, Elliott SJ, et al. (2013) Output power and efficiency of electromagnetic energy harvesting systems with constrained range of motion. Smart Materials and Structures 22(12): 125009.

Hoffstadt T, Graf C and Maas J (2013) Optimization of the energy harvesting control for dielectric elastomer generators. Smart Materials and Structures 22(9): 094028.

$\mathrm{Hu} \mathrm{Y}$, Xue H and $\mathrm{Hu} \mathrm{H}$ (2007) A piezoelectric power harvester with adjustable frequency through axial preloads. Smart Materials and Structures 16(5): 1961-1966.

Institute of Electrical and Electronics Engineers (IEEE) (1988) IEEE standard on piezoelectricity, ANSI-IEEE Std. 176-1987. Available at: http://blogs.cimav.edu.mx/ luis.fuentes/data/files/Curso_Cristalograf\%CC3\%ADa/piezo_ ieee.pdf

Kaltenbacher M (2010) Advanced simulation tool for the design of sensors and actuators. Procedia Engineering 5: 597-600.

Kaltenbacher M (2015) Numerical Simulation of Mechatronic Sensors and Actuators: Finite Elements for Computational Multiphysics. 3rd ed. Berlin: Springer.

Kong N and Ha DS (2012) Low-power design of a selfpowered piezoelectric energy harvesting system with maximum power point tracking. IEEE Transactions on Power Electronics 27(5): 2298-2308.

Lefeuvre E, Audigier D, Richard C, et al. (2007) Buck-boost converter for sensorless power optimization of piezoelectric energy harvester. IEEE Transactions on Power Electronics 22(5): 2018-2025.

Lefeuvre E, Sebald G, Guyomar D, et al. (2009) Materials, structures and power interfaces for efficient piezoelectric energy harvesting. Journal of Electroceramics 22(1-3): 171-179.

Lehoucq RB and Sorensen DC (1996) Deflation techniques for an implicitly restarted Arnoldi iteration. SIAM Journal on Matrix Analysis and Applications 17(4): 789-821.

Lien IC, Shu YC, Wu WJ, et al. (2010) Revisit of series-SSHI with comparisons to other interfacing circuits in piezoelectric energy harvesting. Smart Materials and Structures 19(12): 125009.

Marqui CD, Erturk A and Inman DJ (2010) Piezoaeroelastic modeling and analysis of a generator wing with continuous and segmented electrodes. Journal of Intelligent Material Systems and Structures 21(10): 983-993.

Nelder JA and Mead R (1965) A simplex method for function minimization. Computer Journal 7: 308-313. 
Park J, Lee S and Kwak BM (2012) Design optimization of piezoelectric energy harvester subject to tip excitation. Journal of Mechanical Science and Technology 26(1): 137-143.

PI Ceramic GmbH (2016) P-876 DuraAct power patch transducer. Available at: https://www.piceramic.com/en/products/ piezoceramic-actuators/patch-transducers/p-878-duraactpower-patch-transducer-101795/

Rupitsch SJ and Ilg J (2015) Complete characterization of piezoceramic materials by means of two block-shaped test samples. IEEE Transactions on Ultrasonics, Ferroelectrics, and Frequency Control 62(7): 1403-1413.

Rupitsch SJ and Lerch R (2009) Inverse method to estimate material parameters for piezoceramic disc actuators. Applied Physics A: Materials Science \& Processing 97(4): 735-740.

Shu YC and Lien IC (2006) Analysis of power output for piezoelectric energy harvesting systems. Smart Materials and Structures 15(6): 1499-1512.

Shu YC, Lien IC and Wu WJ (2007) An improved analysis of the SSHI interface in piezoelectric energy harvesting. Smart Materials and Structures 16(6): 2253-2264.

Van Blarigan L, Moehlis J and McMeeking R (2015) Low dimensional modeling of a non-uniform, buckled piezoelectric beam for vibrational energy harvesting. Journal of Smart Materials and Structures 24(6): 065012.

Wang L and Yuan FG (2008) Vibration energy harvesting by magnetostrictive material. Smart Materials and Structures 17(4): 045009.

Wein F, Kaltenbacher M and Stingl M (2013) Topology optimization of a cantilevered piezoelectric energy harvester using stress norm constraints. Structural and Multidisciplinary Optimization 48(1): 173-185.

Wischke M, Masur M, Goldschmidtboeing F, et al. (2010) Electromagnetic vibration harvester with piezoelectrically tunable resonance frequency. Journal of Micromechanics and Microengineering 20(3): 035025.

Wu PH and Shu YC (2015) Finite element modeling of electrically rectified piezoelectric energy harvesters. Smart Materials and Structures 24 (9): 094008.

Xiong X and Oyadiji SO (2014) Modal electromechanical optimization of cantilevered piezoelectric vibration energy harvesters by geometric variation. Journal of Intelligent Material Systems and Structures 25(10): 1177-1195.

Yang Y and Tang L (2009) Equivalent circuit modeling of piezoelectric energy harvesters. Journal of Intelligent Material Systems and Structures 20(18): 2223-2235.

Yiming L, Geng T, Yong W, et al. (2008) Active piezoelectric energy harvesting: general principle and experimental demonstration. Journal of Intelligent Material Systems and Structures 20(5): 575-585.

\section{Appendix I}

In the following, material tensors for PZT-5A are given that were used in section "Influence of coupled mode shapes"

$$
\begin{aligned}
& {\left[\boldsymbol{s}^{\boldsymbol{E}}\right]=\left[\begin{array}{cccccc}
16.4 & -5.74 & -7.22 & 0 & 0 & 0 \\
-5.74 & 16.4 & -7.22 & 0 & 0 & 0 \\
-7.22 & -7.22 & 18.8 & 0 & 0 & 0 \\
0 & 0 & 0 & 47.5 & 0 & 0 \\
0 & 0 & 0 & 0 & 47.5 & 0 \\
0 & 0 & 0 & 0 & 0 & 44.3
\end{array}\right]} \\
& \cdot 10^{-12} \frac{\mathrm{m}^{2}}{\mathrm{~N}} \\
& {[\boldsymbol{d}]=\left[\begin{array}{cccccc}
0 & 0 & 0 & 0 & 584 & 0 \\
0 & 0 & 0 & 584 & 0 & 0 \\
-171 & -171 & 374 & 0 & 0 & 0
\end{array}\right]} \\
& \cdot 10^{-12} \frac{\mathrm{C}}{\mathrm{N}} \\
& {\left[\boldsymbol{\varepsilon}^{\boldsymbol{T}}\right]=\left[\begin{array}{ccc}
1730 & 0 & 0 \\
0 & 1730 & 0 \\
0 & 0 & 1700
\end{array}\right] \cdot \varepsilon_{0}}
\end{aligned}
$$

